\title{
EL DESARROLLO HUMANO \\ COMO LIBERTAD: UNA APROXIMACIÓN A LA PROPUESTA DEL ENFOQUE DE LAS CAPACIDADES DE AMARTYA SEN
}

\author{
Prof. Dra. Vanesa Lorena Battaglino \\ Universidad Nacional de Mar del Plata, Argentina
}

\begin{abstract}
Resumen: El enfoque de las capacidades elaborado por el economista y filósofo Amartya Sen se presenta como un nuevo paradigma teórico en el campo de las políticas públicas y del desarrollo que al evaluar el bienestar de los ciudadanos plantea la siguiente pregunta: ¿qué es capaz de ser y hacer cada persona?, definiendo la calidad de vida en términos de la capacidad para lograr funcionamientos valiosos. En el siguiente trabajo se analiza esta propuesta con el objetivo de mostrar las relaciones que pueden entablarse entre el concepto de libertad defendido por el enfoque y la idea de desarrollo humano, estimando las repercusiones actuales de este marco teórico a nivel internacional.
\end{abstract}

Descriptores: Enfoque de las capacidades $\cdot$ Libertad $\cdot$ Desarrollo humano $\cdot$ Capacidad · Funcionamientos.

\begin{abstract}
The capability approach developed by the economist and philosopher Amartya Sen is introduced as a new theoretical paradigm in the field of public policies and development which, when assessing the citizens' wellbeing, raises the following question: What is each individual able to be and to do when the quality of life is defined in terms of the ability to achieve valuable functionings? In the work described herein below, this proposal is analyzed in order to show the relationship that could be established between the concept of freedom supported by the approach and the idea of human development, taking into account the current impacts of this theoretical approach at international level.
\end{abstract}

Keywords: Capability approach · Freedom ' Human Development · Capability · Functionings.

Enviado: 06/09/2018. Aceptado: 19/12/2018

\section{INTRODUCCIÓN}

La discusión en torno al desarrollo humano y el bienestar se ha vuelto cada vez más habitual no sólo en los análisis filosóficos, económicos y políticos sobre las sociedades, sino también a nivel del discurso común de los ciudadanos. De 
hecho, probablemente la mayoría de las personas podrían, al menos intuitivamente, responder qué implica para cada una de ellas tener una vida humana buena. Y sin dudas un aspecto fundamental de la misma sería poder decidir entre un estilo de vida u otro libremente, valorando así la libertad de elegir y actuar como un fin u objetivo en sí misma y no sólo en un sentido instrumental.

Sin embargo, tradicionalmente la evaluación del bienestar se ha basado en algunos modelos que toman ciertos indicadores indiferenciados -tales como el PBI per cápita, la utilidad o los recursos- que no constituyen la libertad como tal, sino que en general funcionan como medios para propósitos ulteriores. Se trata de modelos que muy poco parecen decir acerca del modo en que las personas viven realmente sus vidas y de lo que ellas valoran como importante, brindando una mirada sesgada al momento de abordar la cuestión del bienestar. Por lo tanto, resulta imprescindible considerar alguna propuesta alternativa que responda de un modo más adecuado a las vidas reales que los individuos son capaces de llevar a cabo y a las decisiones que pueden efectivamente tomar. Tal sería la perspectiva adoptada por el enfoque de las capacidades elaborado por el reconocido economista y filósofo Amartya Sen, cuyos estudios sobre la elección social, la distribución y la pobreza convergen en postular una idea de libertad que sirve de base para las comparaciones interpersonales en la evaluación de los niveles de bienestar; y que cobra relevancia en tanto se vincula con las condiciones o restricciones del espacio de decisión de los individuos.

En este trabajo se propone reflexionar sobre esa idea de libertad a fin de determinar su relación con la noción de desarrollo humano. Para ello se presenta un análisis de los conceptos de capacidad y funcionamiento, señalado sus conexiones con la libertad y planteando la pregunta acerca de cuál de ellos debería ser promovido por los gobiernos como un objetivo político apropiado. Luego se centra la atención en la idea de desarrollo defendida por el enfoque, y a partir de este recorrido se consideran brevemente algunas de las repercusiones del mismo a nivel internacional con el propósito de estimar su influencia y el alcance de sus aportes en los debates actuales sobre el tema.

\section{LA LIBERTAD EN EL ENFOQUE DE LAS CAPACIDADES: ¿Promover capacidades o funcionamientos?}

El trabajo realizado por Amartya Sen se inscribe en el ámbito de la economía del bienestar, su enfoque surgió hacia la década de 1980 como una alternativa a los modelos económico-utilitaristas dominantes que restringen el desarrollo al crecimiento económico. A lo largo de sus investigaciones Sen se ha ocupado de distintas formas de temas como la distribución o la pobreza, reconociendo explícitamente que es la teoría de la elección social (formulada en su forma moderna 
por Kenneth Arrow) la que aporta un enfoque general a la evaluación de, y a la elección entre, posibilidades sociales alternativas (Sen, 1999). Es precisamente desde ese horizonte que conviene llevar a cabo el análisis de su propuesta.

La teoría de la elección social es una disciplina amplia que abarca una variedad de cuestiones diversas, entre las que se incluye el tema de cómo juzgar el bienestar general de una sociedad a partir de la pluralidad de intereses de sus miembros. Desde esta perspectiva Sen plantea la posibilidad de desarrollar una economía del bienestar y una teoría de la elección social constructivas e ir más allá de las reglas de votación (categoría explorada por Borda, Condorcet y Arrow) como base para las evaluaciones del bienestar, de tal forma que puedan abarcarse temas de distribución. La ampliación de la base de información a partir de la cual realizar las evaluaciones supone el uso de comparaciones interpersonales, permitiendo así que las decisiones públicas sean sensibles a las desigualdades en términos de oportunidades y bienestar:

La posibilidad de una economía del bienestar y de una elección social constructivas (y de su uso en la elaboración de evaluaciones del bienestar social en el diseño de medidas prácticas con significado normativo) depende de la necesidad de ampliar la base de información de tales elecciones (...) Un elemento crucial de esta ampliación es el uso de comparaciones interpersonales (...)No podemos siquiera entender la fuerza de las preocupaciones públicas por la pobreza, el hambre, la desigualdad, o la tiranía, sin involucrar algún tipo de comparación interpersonal. La información sobre la cual se fundamentan nuestros juicios informales sobre estos temas es precisamente el tipo de información que debe -y puede- ser incorporada al análisis de la elección social sistemática. (Sen, 1999, p. 365)

En este marco, la pregunta fundamental que se debe responder es la siguiente: ¿comparaciones interpersonales de qué? Cualquier respuesta sugerida demanda la elección de algún tipo de contabilidad de la ventaja individual. Sen argumenta a favor de una evaluación en términos de las capacidades que cada individuo tiene para elegir un modo de vida u otro. De esta forma su enfoque se centra en la libertad, entendida como la capacidad para ser o hacer aquello que se juzga importante, esto es, para lograr funcionamientos valiosos, consagrando así una noción de libertad positiva vinculada con la idea de autodeterminación.

Los conceptos de capacidad y funcionamiento son centrales en la teoría de Sen. A partir de ellos el autor señala que el bienestar de un individuo puede entenderse teniendo en cuenta la calidad de su vida, y la vida es definida como un conjunto de funcionamientos interrelacionados consistentes en acciones y estados. En este contexto la realización de una persona es comprendida como el vector de sus funcionamientos. Los funcionamientos son constitutivos del estado de un individuo, 
específicamente las cosas que logra hacer o ser al vivir, y la evaluación del bienestar consiste en una estimación de esos elementos constitutivos. ${ }^{2}$ Sen $(1995 ; 1996)$ advierte que los funcionamientos pueden abarcar desde cosas muy elementales, como estar bien alimentado o tener buena salud, hasta realizaciones más complejas como tener dignidad o integrarse socialmente.

En estrecha relación con la noción de funcionamientos se encuentra la capacidad de funcionar, que representa las distintas combinaciones de estados y acciones que un individuo puede alcanzar. Por ello se sostiene que la capacidad es un conjunto de vectores de funcionamientos, que reflejan la libertad de una persona para decidir entre distintos modos de vida. La importancia de la capacidad para el bienestar de alguien surge de dos consideraciones distintas pero interrelacionadas. En primer lugar, si los funcionamientos alcanzados constituyen el bienestar de una persona, entonces la capacidad para alcanzar funcionamientos constituirá su libertad, esto es, sus oportunidades reales para obtener dicho bienestar. Por lo tanto, al momento de evaluar una sociedad será necesario tomar en cuenta las libertades de que disfrutan las personas. La segunda relación que puede establecerse entre la capacidad y el bienestar consiste en hacer depender el bienestar alcanzado de la capacidad para funcionar. En este sentido, el hecho de poder decidir/elegir debería ser interpretado como un componente valioso de la existencia. Dado que los individuos son agentes y no meros depositarios del bienestar, Sen defiende que una vida humana buena es una vida rica en decisiones/elecciones valiosas. Por lo tanto, la capacidad de funcionamiento es una parte fundamental de la evaluación social realizada sobre la base de comparaciones interpersonales.

De esta manera el enfoque de las capacidades se concentra en las libertades sustantivas que los individuos tienen y no sólo en los resultados particulares que consiguen alcanzar. Esta concentración en la libertad provee un marco general para analizar la ventaja individual y la privación en las sociedades contemporáneas. En consecuencia, el núcleo del enfoque no es sólo aquello que una persona realmente termina por hacer, sino lo que ella es capaz de hacer, independientemente de que elija o no aprovechar esa oportunidad. ${ }^{3}$ Es por ello que Amartya Sen señala que "al evaluar nuestras vidas, tenemos razón para estar interesados no sólo en la clase de

\footnotetext{
${ }^{2}$ Los fundamentos filosóficos de esta idea se remontan a los escritos de Aristóteles, en los que se encuentra una profunda investigación del bien del hombre en cuanto a la vida entendida como actividad. Para un análisis del planteamiento aristotélico y sus relaciones con la perspectiva de la capacidad véase Nussbaum (1988).

${ }^{3}$ Esta característica del enfoque ha sido cuestionada por varios autores, entre ellos Richard Arneson (1989) y Gerald Cohen (1996), quienes han formulado argumentos a favor de la relevancia de la realización efectiva de actividades, en lugar de poner la atención en la capacidad para elegir entre diferentes realizaciones.
} 
vidas que conseguimos vivir, sino también en la libertad que realmente tenemos para escoger entre diferentes estilos y modos de vida" (Sen, 2011, p. 257).

La libertad es valiosa por dos razones principales. En primer lugar, porque brinda más oportunidad de perseguir los propios objetivos, es decir, aquello que se estima importante para la propia vida. Por lo tanto, sirve a la habilidad para decidir cómo se quiere vivir y promover los fines que se desean impulsar; ese es el denominado "aspecto de oportunidad" de la libertad. En segundo lugar, también se puede atribuir importancia al proceso de elección como tal; ese es el denominado "aspecto de proceso". El concepto de capacidad se vincula estrechamente con el aspecto de oportunidad, de tal manera que la ventaja general de una persona se juzga menor que la de otra si tiene menos capacidad/oportunidad real de lograr aquellas cosas que tenga razón para valorar.

Amartya Sen (2005, pp. 152-153; 2011, pp. 259-260) ilustra esta distinción mediante el siguiente ejemplo: Kim decide un domingo que prefiere quedarse en su casa en lugar de salir. Si consigue hacer exactamente lo que desea se encuentra en el escenario A. Pero unos maleantes armados interrumpen su descanso y lo sacan a la fuerza de su casa arrojándolo a una cuneta. Esta situación es el escenario B. En una tercera instancia, el escenario $\mathrm{C}$, los maleantes someten a Kim ordenándole no salir de su casa bajo amenazas. Es evidente que en el escenario B la libertad de Kim resulta afectada, puesto que no puede hacer lo que quería y su libertad de decidir por sí mismo desaparece. Por lo tanto, queda violado el aspecto de oportunidad de la libertad del protagonista como también el de proceso. En el escenario $\mathrm{C}$ el aspecto de proceso de la libertad es claramente afectado, porque no podría haber hecho nada distinto sin ser castigado por ello. Lo interesante aquí es lo concerniente al aspecto de oportunidad. Dado que Kim hace lo mismo en los escenarios A y C, bajo amenazas o no, la pregunta que se plantea es la siguiente ¿podría afirmarse que su aspecto de oportunidad es el mismo en ambos? Si la oportunidad que la persona tiene se juzga sólo considerando si termina haciendo lo que escogería hacer sin estar bajo amenazas, entonces no hay diferencia entre los escenarios A y C. El aspecto de oportunidad de la libertad no resulta alterado bajo esta visión estrecha. Pero el enfoque propone una concepción más amplia que permite sostener que en el escenario $\mathrm{C}$ el aspecto de oportunidad de Kim también resulta socavado porque se le ordena permanecer en su casa sin poder elegir otras alternativas.

El vínculo entre las capacidades y los funcionamientos es quizás más complejo de lo que puede parecer a primera vista. Sen (1996) establece que se trata de una relación bidireccional y simultánea. Al comparar los dos conceptos hay que considerar que capacidad significa "oportunidad de seleccionar", es decir que la noción de libertad de elección o de decisión está inscripta en la idea misma de capacidad. A modo de ejemplo, una persona que pasa hambre y otra que ayuna 
tienen el mismo funcionamiento en lo que concierne a la nutrición, sin embargo no disponen de la misma capacidad; quien ayuna es capaz de no ayunar (se trata de una decisión que podría o no haber tomado), mientras que la persona hambrienta lo es porque no tiene elección (Sen, 2011).

Por lo tanto, las capacidades son libertades sustanciales, un conjunto de oportunidades generalmente interrelacionadas para elegir y actuar. Martha Nussbaum, quien ofrece una versión normativa del enfoque, incorpora el concepto de capacidades combinadas para referirse a ellas. Esta noción alude a la totalidad de oportunidades de las que dispone una persona para elegir y actuar en su situación política, social y económica concreta. Las capacidades combinadas se distinguen de las capacidades internas, entendidas como ciertas características o estados fluidos y dinámicos de una persona, tales como los rasgos de su personalidad, sus capacidades intelectuales y emocionales, su estado de salud, su aprendizaje interiorizado, y en general aquellos rasgos y aptitudes entrenados y desarrollados en interacción con el entorno. Si bien dichas características son sumamente importantes para las capacidades combinadas, es preciso diferenciarlas comprendiendo a estas últimas como "la suma de las capacidades internas y las condiciones sociales/políticas/ económicas en las que puede elegirse realmente el funcionamiento de aquellas" (Nussbaum, 2012, p. 42). Es necesario advertir que la distinción entre capacidades internas y combinadas no es diáfana, puesto que una persona adquiere usualmente una capacidad interna gracias a cierta forma de funcionamiento y puede perderla si no tiene la oportunidad de funcionar. A pesar de esto, conviene mantener la diferenciación porque constituye una especie de método heurístico útil para diagnosticar las deficiencias y los logros en una sociedad determinada.

Teniendo en cuenta la relevancia de los conceptos de capacidad y funcionamiento y su relación con la idea de libertad, la pregunta ineludible que surge a esta altura del análisis es si, en términos de objetivos políticos, deben promoverse capacidades o funcionamientos. Se trata de un asunto controvertido sobre el que puede no haber unanimidad de criterio. Para esbozar una respuesta es necesario tener en cuenta que en cierta forma las capacidades son importantes porque se traducen en funcionamientos, puesto que si las personas nunca funcionaran no sería sensato considerar que su sociedad es buena o justa simplemente porque les ha conferido muchas capacidades. Pero las capacidades también tienen valor en sí mismas como ámbitos de libertad y elección, esto es, como las libertades sustantivas para alcanzar combinaciones alternativas de funcionamientos. Por lo tanto, fomentar capacidades es fomentar áreas de libertad, lo que difiere considerablemente de hacer que las personas funcionen de una determinada manera. De modo que, los objetivos políticos apropiados de los gobiernos deberían ser las capacidades y no los funcionamientos, porque son las primeras las que garantizan la existencia de 
un espacio para el ejercicio de la libertad de decisión. Hay una diferencia moral muy grande entre una política que promueve funcionamientos y otra que fomenta las capacidades, puesto que la segunda (y no la primera) es la que efectivamente respeta la elección de estilo de vida de la persona. Si bien hay que reconocer que son los funcionamientos, y no simplemente las capacidades, los que tornan una vida como verdaderamente humana, para propósitos políticos lo apropiado es ir tras las capacidades.

De esto se sigue que las capacidades son prioritarias sobre los funcionamientos, pues constituyen la base de alternativas de decisión. En este sentido podría afirmarse que la capacidad para funcionar añade a la idea de funcionamiento un elemento fundamental: la posibilidad real de decidir o no funcionar de una determinada manera (Cejudo, 2007). Sin embargo, algunos autores que emplean el enfoque consideran que en ciertas áreas el Estado está legitimado para promover funcionamientos; tal es el caso de Richard Arneson, quien defiende políticas de orientación paternalista en el ámbito de la salud, considerando que los gobiernos deberían utilizar su poder para hacer que las personas adopten efectivamente estilos de vida sanos. Pero tanto Sen como Nussbaum discrepan con esta postura debido al elevado valor que le atribuyen a la libertad de elección. Por otro lado, para realizar evaluaciones sociales referidas al bienestar y al desarrollo, son las capacidades las que permiten una extensión mayor de la base de la información al momento de llevar a cabo comparaciones interpersonales. El marco de la capacidad se presenta, entonces, como el espacio idóneo para realizar comparaciones sobre la calidad de vida.

\section{El DESARROLLO HUMANO EN EL ENFOQUE DE LAS CAPACIDADES}

En consonancia con el lugar prioritario que se le atribuye a la libertad para realizar evaluaciones del bienestar, el enfoque de las capacidades establece una conexión fundamental entre ella y el desarrollo humano, asumiendo como punto de partida la identificación de la libertad como el objetivo principal del desarrollo. Así, este último es definido como "un proceso de expansión de las libertades reales de que disfrutan los individuos" (Sen, 2000, p. 19).

Esta concepción contrasta con las visiones más estrictas del desarrollo, que lo identifican con el crecimiento del PBI, con el aumento de la renta personal, con los avances tecnológicos, con la modernización social o con la industrialización. Si bien el crecimiento del PBI o de la renta puede ser un medio importante para expandir las libertades, lo cierto es que éstas también dependen de otros determinantes, como por ejemplo de los derechos políticos y humanos o de las instituciones sociales y económicas. Asimismo, el progreso tecnológico, la modernización social y la industrialización pueden contribuir significativamente en la expansión de la libertad 
de los individuos, pero esta última requiere además de otros factores. Es por ello que Amartya Sen sostiene que si lo que promueve el desarrollo es la libertad, entonces existen poderosos argumentos para concentrar los esfuerzos en ese objetivo general y no en algunos medios o instrumentos especialmente elegidos (Sen, 2000). Por lo tanto, la concepción del desarrollo como un proceso de expansión de las libertades fundamentales lleva a poner la atención en los fines por los que cobra importancia el desarrollo, y no solamente en los medios que desempeñan un papel destacado en dicho proceso. Por esta razón suele afirmarse que el enfoque de las capacidades implica un cambio fundamental en el foco de atención: de los medios de vida a las oportunidades reales de vivir que tienen las personas (Sen, 2011).

Para ilustrar el carácter radical de la idea del desarrollo como libertad Sen (2000, pp. 21-22) propone pensar en tres ejemplos elementales. El primero de ellos apunta a mostrar que en las visiones en las que se identifica al desarrollo con el crecimiento económico, por ejemplo en el enfoque del PBI, a menudo se pregunta si algunas libertades sociales o políticas (libertad de participación política, oportunidad de recibir una educación básica, etc.) contribuyen o no al desarrollo. Pero esta manera de plantear la pregunta tiende a pasar por alto que esas libertades fundamentales se encuentran entre los componentes constitutivos del desarrollo, lo que supone que su importancia no debe demostrarse a través de su contribución directa al crecimiento económico. El segundo ejemplo señala la discordancia que puede haber entre la renta per cápita y la libertad de las personas para vivir una vida humana buena y larga. Así, es posible que los ciudadanos de Namibia o Gabón sean mucho más ricos desde el punto de vista del PBI per cápita que los de Sri Lanka o Kerala, pero la esperanza de vida de los segundos es superior a la de los primeros. En tercer lugar el autor plantea que frecuentemente se afirma que los afroamericanos que viven en Estados Unidos son relativamente pobres en comparación con los estadounidenses blancos, pero más ricos que las personas del Tercer Mundo. Sin embargo, Sen advierte que los afroamericanos tienen menos probabilidades en términos absolutos de llegar a la edad adulta que los habitantes de muchos países del Tercer Mundo, como Sri Lanka o algunas partes de India. La presencia de estos contrastes entre diferentes grupos dentro de los países más ricos es un aspecto importante que hay que tener en cuenta para comprender lo que implican el desarrollo y el subdesarrollo en términos de libertad.

Ahora bien, en el marco de este enfoque la expansión de la libertad, además de ser el fin del desarrollo, también es su medio. Esto supone que, junto con el reconocimiento de la importancia central de la libertad en la realización de evaluaciones, también es necesario resaltar la relación empírica que hay entre los distintos tipos de libertades. En ese sentido, la eliminación de algunos tipos de falta de libertad que dejan a los individuos pocas opciones y oportunidades para 
ejercer su agencia razonada, se configura como un aspecto central del desarrollo. Igualmente adquiere relevancia la eficacia instrumental de algunos tipos de libertad para fomentar otros como un complemento poderoso de la importancia intrínseca de la libertad de las personas en general. Sobre este tema es interesante señalar que el enfoque se ha visto enriquecido desde hace algunos años con los aportes de Jonathan Wolff y Avner De-Shalit (2007), quienes proponen los conceptos de capacidad fértil y desventaja corrosiva para dar cuenta de dicha eficacia instrumental. El primero alude a aquellas capacidades que tienden a favorecer la presencia de otras, un ejemplo de esto sería el caso de la educación, que abre opciones de muchas clases. Las desventajas corrosivas constituyen privaciones de algunas libertades que tienen efectos muy significativos en diversas áreas de la vida de las personas, repercutiendo en la falla de otras capacidades; la estigmatización y la discriminación serían fuentes de las mismas. Investigar posibles capacidades fértiles y desventajas corrosivas contribuye a ampliar la información para realizar evaluaciones del bienestar y, consecuentemente, detectar situaciones donde podrían intervenir más adecuadamente las políticas públicas, por lo que sin dudas se configura como una tarea indispensable en toda sociedad que aspire a promover el desarrollo de los ciudadanos. $^{4}$

Por otra parte, es importante señalar que la libertad es esencial para el desarrollo por dos motivos distintos. En primer lugar, por la evaluación. Esto implica que el progreso ha de evaluarse en función del aumento que hayan experimentado o no las libertades de las personas. Aquí hay que considerar que el hecho de tener más libertad para hacer aquello que se valora es importante por derecho propio para la libertad total de la persona, pero también es relevante para aumentar las oportunidades de los individuos con vistas a obtener resultados valiosos. En segundo lugar, la libertad también es esencial para el desarrollo por la eficacia. Esto supone que la libertad no sólo es la base de la evaluación del éxito y del fracaso, sino también un determinante de la iniciativa individual y de la eficacia social. De tal manera que el aumento de la libertad mejora la capacidad de los individuos para ayudarse a sí mismos como así también para influir en el mundo, y esto es fundamental para el proceso de desarrollo. En este sentido Sen sostiene que el desarrollo depende totalmente de la libre agencia de los individuos, es decir, de su capacidad para elegir y perseguir las propias metas. Esto implica ciertas conexiones entre los distintos

\footnotetext{
${ }^{4}$ Otro aporte relevante al enfoque realizado por Wolff y De-Shalit es la noción de "seguridad de la capacidad". Esto supone que las personas no sólo necesitan tener una capacidad hoy, sino también una expectativa asegurada de que seguirán teniendo esa capacidad mañana. Por lo tanto, se considera que las políticas públicas no deben limitarse a promover capacidades, sino que deben hacerlo de tal manera que las personas puedan contar con ellas de cara al futuro. Estos autores han trabajado en Inglaterra y en Israel con colectivos de inmigrantes y han descubierto que la seguridad acerca del futuro es sumamente importante en relación a la posibilidad real de disfrutar de las libertades.
} 
tipos de libertad que se refuerzan mutuamente. Son esas interconexiones las que hacen que la libre agencia constituya un importante motor del desarrollo. ${ }^{5}$

Para cerrar este análisis es interesante considerar que la idea de que el desarrollo consiste en una ampliación de las libertades podría correr el riesgo de caer en una generalidad abstracta si no se establece de alguna manera cuáles serían esas libertades/capacidades. Sin embargo Sen no especifica un catálogo cerrado de capacidades necesarias para alcanzar una vida humana buena. Desde su punto de vista la determinación exacta de todas las capacidades básicas no es necesaria en la práctica, puesto que bastan las diferencias en una o en algunas de ellas para señalar desigualdades relevantes en los niveles de vida.

Martha Nussbaum, en cambio, elabora una lista concreta de diez capacidades centrales que se configuran como un umbral por debajo del cual no es posible alcanzar una vida digna. En ella incluye: vida; salud física; integridad física; sentidos, imaginación y pensamiento; emociones; razón práctica; afiliación; otras especies; control sobre el propio entorno político y material (Nussbaum, 2002, pp. $120-123 ; 2007$, pp. $88-89 ; 2012$, pp. 53-55). Esta es una importante diferencia entre la versión normativa del enfoque elaborada por Nussbaum, que se orienta a la construcción de una teoría de la justicia social básica, y la versión comparativa de Sen, que se centra en el uso comparativo de las capacidades para evaluar la calidad de vida. A pesar de las diferencias, ambas versiones podrían ser interpretadas como complementarias, en tanto comparten las nociones esenciales y la idea fundamental del desarrollo de los individuos y de los países en términos de las libertades para decidir en los ámbitos sociales, políticos y económicos.

\section{ALGUNAS REPERCUSIONES DEL ENFOQUE A NIVEL INTERNACIONAL}

A nivel internacional puede advertirse una creciente influencia del enfoque de las capacidades en las organizaciones que debaten cuestiones vinculadas con el bienestar y el desarrollo. En este sentido, bajo el liderazgo intelectual de Mahbub ul Haq, desde el año 1990 el Programa de las Naciones Unidas para el Desarrollo (en adelante PNUD) ha hecho un uso sistemático de un tipo particular de ampliación de la base de información para hacer comparaciones basadas en las características observadas en las condiciones de vida de los individuos, influenciado fuertemente por la propuesta de Sen.

\footnotetext{
${ }^{5}$ El uso que hace este enfoque del término "agencia" merece algunas aclaraciones. La expresión "agente" es utilizada generalmente en la literatura sobre economía y sobre la teoría de los juegos para hacer referencia a una persona que actúa en representación de otra. En cambio Sen lo emplea en el sentido más antiguo de la persona que actúa y provoca cambios, y cuyos logros pueden juzgarse en función de sus propios objetivos y valores, independientemente de que se evalúen o no también en función de ciertos criterios externos.
} 
En este paradigma el desarrollo se analiza y concibe poniendo en el centro de atención la posibilidad de elección/decisión de las personas. Esto supone un fuerte contraste con las visiones anteriores que al momento de analizar el desarrollo se enfocaban exclusivamente en el ingreso. De esta forma se sostiene que el propósito básico del desarrollo humano es ampliar las opciones de las personas, creando un ambiente que les permita disfrutar de una vida larga, saludable y creativa (Haq, 1995). Este modelo lleva a cabo un importante aporte al cuestionar el supuesto vínculo automático entre la ampliación del ingreso y la ampliación de las opciones humanas, lo que no significa rechazar que el crecimiento económico sea un factor relevante para el desarrollo. De lo que se trata es de postular que el horizonte de las políticas de desarrollo es mejorar las vidas de las personas y no sólo la ampliación de los procesos productivos.

Asimismo, este paradigma defiende que el desarrollo humano abarca dos aspectos importantes. Por una parte, el fomento de capacidades humanas, y por otro lado el uso que las personas hacen de ellas. Además, mantiene una cuidadosa distinción entre medios y fines, lo que implica que las personas son consideradas como fines, pero sin desatender el tema de los medios. De forma tal que la ampliación del PBI se convierte en un medio necesario para ampliar las opciones de los individuos, pero el carácter y la distribución del crecimiento económico son medidos a partir del criterio de enriquecer sus vidas. Por lo tanto, lo decisivo es el uso que las personas hacen de la riqueza y no la riqueza en sí misma, estimando que aquello que enriquece una vida humana no es el ingreso en sí, sino las oportunidades para decidir y hacer elecciones valiosas.

En consecuencia, se propone una noción de desarrollo humano como un paradigma de desarrollo holístico que abarca tanto medios como fines, productividad e igualdad, desarrollo social y económico, bienes materiales y bienestar humano. Los cuatro pilares fundamentales del desarrollo humano serían los siguientes: igualdad, sustentabilidad, productividad y empoderamiento. Se considera que el crecimiento económico es fundamental, pero a la vez se enfatiza la necesidad de poner atención a su calidad y distribución; se analiza detalladamente su vínculo con las vidas de las personas en tanto agentes y no meros receptores pasivos; y se cuestiona su sustentabilidad a largo plazo (Haq, 1995).

Sobre la base de estas consideraciones, en los informes sobre desarrollo humano publicados anualmente se clasifica a las naciones en función del Índice de Desarrollo Humano (en adelante IDH), que evalúa el bienestar a partir de tres dimensiones básicas: a) La esperanza de vida al nacer, que refleja la capacidad de llevar una vida larga y saludable; b) Los años promedio de escolaridad y los años esperados de escolaridad, que reflejan la capacidad de adquirir conocimientos; y c) El ingreso nacional bruto per cápita, que refleja la capacidad de lograr un nivel de 
vida decente. Además, para medir el desarrollo de un modo más completo se tienen en cuenta cuatro índices compuestos: a) El IDH ajustado por la desigualdad, que descuenta al mismo en función de la magnitud de la desigualdad; b) El Índice de Desarrollo de Género, que compara los valores del IDH para mujeres y hombres; c) El Índice de Desigualdad de Género, que hace hincapié en el empoderamiento de las mujeres; y d) El Índice de Pobreza Multidimensional, que mide las dimensiones de la pobreza no referidas a los ingresos (PNUD, 2015a, p. 3).

De esta manera el desarrollo humano es medido a partir de un criterio amplio que no se restringe sólo al crecimiento económico, sumando dimensiones que alejan a esta perspectiva de los enfoques dominantes y la acercan significativamente a la propuesta del enfoque de las capacidades. A modo de ejemplo, puede mencionarse que en el Informe sobre Desarrollo Humano 2016 se apunta al desarrollo para todos, señalando que el mismo tiene por objeto las libertades humanas. En este documento la referencia a algunos conceptos centrales del enfoque de las capacidades -tales como capacidad, funcionamiento y libertad- para definir el desarrollo es explícita:

El desarrollo humano consiste en ampliar las libertades de modo que todos los seres humanos puedan aprovechar las posibilidades que consideren más valiosas. Estas libertades tienen dos aspectos fundamentales: la libertad de bienestar, representada por los funcionamientos (functionings) y las capacidades (capabilities), y la libertad de agencia (freedom of agency), representada por la voz y la autonomía (...) Ambos tipos de libertades son absolutamente necesarios para el desarrollo humano. (PNUD, 2016, pp. 1-2)

Allí también se especifica la idea de que la libertad es un medio para ampliar las oportunidades pero a la vez es el objetivo mismo del desarrollo, dando cuenta de la amplitud que define al enfoque adoptado y distinguiéndolo de otros modelos:

El desarrollo humano es un proceso encaminado a ampliar las oportunidades de las personas. Pero también es un objetivo, por lo que constituye a la vez un proceso y un resultado. El desarrollo humano implica que las personas deben influir en los procesos que determinan sus vidas. En este contexto, el crecimiento económico es un medio importante para el logro del desarrollo humano, pero no es el fin último (...) Se trata de un enfoque más amplio que otros, como el enfoque de recursos humanos, el de necesidades básicas y el de bienestar humano. (PNUD, 2016, p. 2)

A partir de esta concepción general, en las discusiones más recientes se ha ampliado la idea de desarrollo abarcando las dimensiones sociales, económicas y ambientales condensadas en la idea de desarrollo sostenible, que hoy se ha convertido en el eje principal del debate. Consecuentemente, se ha elaborado la Agenda 2030 para el Desarrollo Sostenible, aprobada por la comunidad internacional en septiembre de 
2015 durante el septuagésimo período de sesiones de la Asamblea General de las Naciones Unidas. ${ }^{6}$ En ella se proponen 17 objetivos de desarrollo sostenible y 169 metas, con los que se pretende retomar los Objetivos de Desarrollo del Milenio y conseguir lo que éstos no lograron. ${ }^{7}$

La idea de desarrollo sostenible lleva implícitas las nociones de responsabilidad, solidaridad y libertad. Es un principio de organización del desarrollo mundial que vela por el bienestar tanto de las personas como del planeta y se configura como un asunto de justicia social relacionado con la equidad intergeneracional, en tanto implica las libertades de las generaciones futuras y presentes (PNUD, 2016). Desde su aparición este concepto se ha extendido para propiciar un acercamiento entre las preocupaciones sociales, medioambientales y económicas, con la intención de aunar el desarrollo humano y social, la protección del medio ambiente y la cuestión económica.

De esta manera el concepto de desarrollo como expansión de las libertades es ampliado y enriquecido sumando otros aspectos relevantes e incluyendo a las generaciones por venir. El espíritu que anima la Agenda 2030 se asemeja significativamente a los principios y demandas generales impulsados por la perspectiva de la capacidad. Así, se declara el respeto por la libertad y la dignidad de las personas, se afirma la necesidad de lograr el empoderamiento de los más vulnerables y se postula el compromiso de propiciar la plena realización de las capacidades, reconociendo la diversidad que caracteriza a la vida humana:

Todas las personas, sea cual sea su sexo, raza u origen étnico, incluidas las personas con discapacidad, los migrantes, los pueblos indígenas, los niños y los jóvenes,

\footnotetext{
${ }^{6}$ Desde el Informe Brundtland de 1987, Nuestro futuro común, en el que se popularizó la noción más común de desarrollo sostenible, tres reuniones internacionales desempeñaron un papel decisivo en la elaboración de esta Agenda: la Conferencia de las Naciones Unidas sobre el Medio Ambiente y el Desarrollo (también conocida como Cumbre para la Tierra) en el año 1992, la Cumbre Mundial sobre el Desarrollo Sostenible celebrada en Johannesburgo en 2002 y La Conferencia de las Naciones Unidas sobre el Desarrollo Sostenible celebrada en Río de Janeiro en 2012. En el Informe Brundtland se enumeraron los siguientes objetivos críticos vinculados con el desarrollo sostenible: cambiar la calidad del crecimiento económico; satisfacer las necesidades básicas en materia de trabajo, alimentos, energía, agua e higiene; lograr un nivel de población aceptable; conservar y acrecentar los recursos naturales; reorientar la tecnología y controlar los riesgos; tener en cuenta el medio ambiente y la economía en la adopción de decisiones; y reorientar las relaciones económicas internacionales para construir un desarrollo más participativo. Desde entonces la comprensión del desarrollo sostenible ha evolucionado derivando en la Agenda 2030 para el Desarrollo Sostenible (UNESCO, 2017).

${ }^{7}$ Los Objetivos de Desarrollo del Milenio se presentaron como ocho propósitos de desarrollo humano en el año 2000, que los países miembros de las Naciones Unidas acordaron alcanzar para el año 2015. Tales objetivos fueron enumerados de la siguiente manera: 1) Erradicar la pobreza extrema y el hambre; 2) Lograr la enseñanza primaria universal; 3) Promover la igualdad de género y la autonomía de la mujer; 4) Reducir la mortalidad infantil; 5) Mejorar la salud materna; 6) Combatir el VIH/SIDA, paludismo y otras enfermedades; 7) Garantizar la sostenibilidad del medio ambiente; y 8) Fomentar una asociación mundial para el desarrollo (Naciones Unidas, 2015a).
} 
especialmente si se encuentran en situaciones de vulnerabilidad, deben tener acceso a posibilidades de aprendizaje permanente que las ayuden a adquirir los conocimientos y aptitudes necesarios para aprovechar las oportunidades que se les presenten y participar plenamente en la sociedad. Nos esforzaremos por brindar (...) un entorno propicio para la plena realización de sus derechos y capacidades. (Naciones Unidas, 2015b, p. 8)

De modo que, en un momento en el que la comunidad internacional ha definido una nueva agenda de desarrollo, este enfoque sigue siendo sumamente útil para articular los objetivos del desarrollo y mejorar el bienestar de las personas asegurando la sostenibilidad.

Por otra parte, también puede observarse la influencia de este paradigma en el Informe de la Comisión sobre la Medición del Desarrollo Económico y del Progreso Social, conocido popularmente como el Informe de la Comisión Sarkozy. El mismo fue elaborado a pedido del entonces presidente de Francia, Nicolás Sarkozy. Insatisfecho con el estado de la información estadística sobre economía y sociedad, en febrero de 2008 Sarkozy solicitó a los economistas Joseph Stiglitz (presidente de la comisión), Amartya Sen (consejero) y Jean Paul Fitoussi (coordinador) que conformaran una comisión con los siguientes objetivos: determinar los límites del PBI como indicador de los resultados económicos y del progreso social; reexaminar los problemas relativos a la medición; identificar los datos adicionales que podrían ser necesarios para obtener indicadores del progreso social más pertinentes; evaluar la viabilidad de nuevos instrumentos de medición; y debatir sobre la presentación adecuada de los datos estadísticos. El resultado de este trabajo fue la elaboración del informe cuya síntesis fue presentada en septiembre de 2009.

En este documento se cuestionan los alcances del PBI como indicador del progreso social y se evalúa la viabilidad de otros instrumentos de medición más adecuados. De esta manera se pretende argumentar que el progreso social no debe ser definido estrictamente sólo en términos de crecimiento económico, reconociendo la importancia de tomar en consideración las oportunidades reales de las personas y su libertad de elección, ampliando así la base de información al momento de hacer evaluaciones sociales y económicas:

La calidad de vida depende de las condiciones objetivas en las cuales se encuentran las personas y de sus capacidades dinámicas (...) Las informaciones que permiten evaluar la calidad de vida (...) incluyen también la medida de sus funcionamientos (...) y de sus libertades. Lo que importa realmente, en efecto, son las capacidades de las cuales disponen las personas, es decir, el conjunto de posibilidades que se ofrecen a ellas y su libertad de escoger, en este conjunto, el tipo de vida al cual otorgan valor. (Stiglitz, Sen \& Fitoussi, 2009, p. 13) 
Otra muestra del cada vez mayor influjo de este enfoque es el destacado trabajo realizado por la Asociación sobre Desarrollo Humano y Capacidades (Human Development and Capability Association), que reúne alrededor de 700 miembros de 70 países. Se trata de una comunidad global de profesionales y académicos dedicada a estudiar la idea de desarrollo humano y el enfoque de las capacidades con la intención de relacionarlos con el ámbito de las políticas. Esta Asociación se orienta fundamentalmente a la promoción de investigaciones de calidad en una amplia variedad de disciplinas (filosofía, economía, salud, educación, derecho, sociología, etc.), contribuyendo así a la discusión teórica y brindando nuevas categorías de análisis para pensar diversas problemáticas vinculadas con el desarrollo y el fomento de capacidades. ${ }^{8}$

Lo que estos ejemplos ponen de manifiesto es que la concepción del desarrollo en términos de libertad propugnado por el enfoque de las capacidades ha ganado terreno en las discusiones internacionales actuales, por lo que dicha teoría parece configurarse como una propuesta mejor equipada que los modelos dominantes para responder a los problemas y desafíos del mundo contemporáneo en materia de desarrollo humano.

\section{CONSIDERACIONES FINALES}

El análisis realizado sobre el concepto de libertad propugnado por el enfoque de las capacidades ha permitido mostrar que el mismo se inscribe en el marco general de la teoría de la elección social, y es desde esta perspectiva más amplia que pueden evaluarse sus alcances, como así también sus relaciones con las nociones centrales de la teoría de Sen y determinar la posibilidad de establecer algún índice de medición del desarrollo. En este sentido, la propuesta de Sen apunta a desarrollar una economía del bienestar y una teoría de la elección social constructivas, defendiendo que al ampliar la base de información es posible disponer de unos criterios coherentes y compatibles para hacer valoraciones sociales y económicas.

La ventaja de utilizar la perspectiva de la capacidad para realizar valoraciones $\mathrm{y}$ evaluaciones radica en que las funciones individuales pueden prestarse con más facilidad a una comparación interpersonal que cualquier comparación de las utilidades. Además, esta perspectiva es inevitablemente pluralista, y eso representa una ventaja ante la reducción radical del razonamiento valorativo que supone el hecho de establecer una magnitud homogénea.

Por otra parte, el recorrido propuesto ha posibilitado comprender que la reorientación asumida por el enfoque hacia el concepto de libertad/oportunidad

\footnotetext{
${ }^{8}$ Para consultar el trabajo realizado por esta Asociación véase https://hd-ca.org/
} 
real/capacidad suministra una guía más clara que otras alternativas para sopesar las prioridades de cualquier política orientada a hacer frente a las desventajas en pos de mejorar la calidad de vida de los ciudadanos. Al tratarse tanto de una teoría de la justicia social básica como de una descripción comparativa de la calidad de vida, sirve en buena medida para remediar algunas de las deficiencias de los modelos dominantes. A diferencia de ellos, parte de un fuerte compromiso con la igual dignidad de todos, siendo sensible a la diversidad cualitativa y a la complejidad de los objetivos que persiguen las personas y también a la distribución, centrándose así en las luchas de los sectores tradicionalmente excluidos. Asimismo, puede tener en cuenta, entre otras cosas, el interés del utilitarismo por el bienestar de las personas, la preocupación del pensamiento libertario por los procesos de elección y por la libertad para actuar y el énfasis de la teoría de John Rawls en la libertad individual $\mathrm{y}$ en los recursos para disfrutar de las libertades fundamentales. Por lo tanto, su amplitud y sensibilidad determinan su enorme alcance y le permiten valorar una extensa variedad de aspectos importantes, algunos de los cuales son dejados de lado por otros enfoques. Este alcance es posible porque las libertades de las personas pueden juzgarse haciendo referencia explícita a los resultados y los procesos que tienen razones para perseguir y valorar. Es justamente la combinación de un análisis fundamental y de un uso pragmático lo que posibilita que esta perspectiva tenga un alcance amplio.

Por estos motivos ciertamente la perspectiva del enfoque elaborado por Amartya Sen ha ganado terreno y se observa que, a pesar de su juventud, este nuevo paradigma teórico puede hacer contribuciones significativas al debate contemporáneo sobre la evaluación y medición del desarrollo.

\section{BIBLIOGRAFÎA}

Arneson, R. (1989). "Equality and Equal Opportunity for Welfare”. Philosophical Studies, Vol. 56, 1, pp. 77-93.

Cejudo, R. (2007). "Capacidades y libertad. Una aproximación a la teoría de Amartya Sen”. Revista Internacional de Sociología, Vol. 65, 47, pp. 9-22.

Cohen, G. (1996). "¿Igualdad de qué? Sobre el bienestar, los bienes y las capacidades". En M. Nussbaum \& A. Sen (Comps.). La calidad de vida (pp. 27-53). México: Fondo de Cultura Económica.

Haq, M. (1995). Reflections on human development. New York: Oxford University Press.

Naciones Unidas (2015a). Objetivos de Desarrollo del Milenio. Informe de 2015. [Versión digital]. Recuperado de http://www.un.org/es/millenniumgoals/pdf/2015/mdg-report-2015 spanish.pdf 
Naciones Unidas (2015b). Resolución aprobada por la Asamblea General el 25 de septiembre de 2015. Transformar nuestro mundo: la Agenda 2030 para el Desarrollo Sostenible. [Versión digital].Recuperado de http://unctad.org/meetings/es/ SessionalDocuments/ares70d1_es.pdf

Nussbaum, M. (1988). "Nature, Function and Capability: Aristotle on Political Distribution”. Oxford Studies in Ancient Philosophy: Supplementary Volume, pp. 145-184.

Nussbaum, M. (2002). Las mujeres y el desarrollo humano. El enfoque de las capacidades. Barcelona: Herder.

Nussbaum, M. (2007). Las fronteras de la justicia. Consideraciones sobre la exclusión. Barcelona: Paidós

Nussbaum, M. (2012). Crear capacidades. Propuesta para el desarrollo humano. Barcelona: Paidós.

PNUD (2015a). Informe sobre Desarrollo Humano 2015. Trabajo al servicio del desarrollo humano. [Versión digital]. Recuperado de: http://hdr.undp.org/sites/default/files/2015_human development_report_overview_-_es.pdf

PNUD (2015b). Transformar nuestro mundo: la Agenda 2030 para el Desarrollo Sostenible. [Versión digital]. Recuperado de: http://www.ar.undp.org/content/dam/argentina/Publications/ Agenda2030/PNUDArgent-DossierODS.pdf

PNUD (2016). Informe sobre Desarrollo Humano 2016. Desarrollo humano para todos. [Versión digital]. Recuperado de: http://hdr.undp.org/sites/default/files/HDR2016_SP_ Overview_Web.pdf

Sen, A. (1983). "Development: Which Way Now? The Economic Journal, Vol. 93, № 372, pp. 745-762. Recuperado de http://darp.lse.ac.uk/PapersDB/Sen_(EJ_83).pdf

Sen, A. (1994). “¿Igualdad de qué?”. En J. Rawls, A. Sen \& otros. Libertad, igualdad y derecho. Las Conferencias Tanner sobre Filosofía Moral (pp. 133-156). Barcelona: Ed. Planeta DeAgostini.

Sen, A. (1995). Nuevo examen de la desigualdad. Madrid: Alianza.

Sen, A. (1996). “Capacidad y bienestar”. En M. Nussbaum \& A. Sen (Comps.). La calidad de vida (pp. 54-83). México: Fondo de Cultura Económica.

Sen, A. (1997). Bienestar, justicia y mercado. Barcelona: Paidós.

Sen, A. (1999). "The Possibility of Social Choice." American Economic Review, Vol. 89, $\mathrm{N}^{o} 3$, pp. 349-378. Recuperado de http://mikael.cozic.free.fr/sen99.pdf

Sen, A. (2000). Desarrollo y libertad. Barcelona: Planeta.

Sen, A. (2005). "Human Rights and Capabilities". Journal of Human Development and Capabilities, Vol. 6, No 2, pp. 151-166. Recuperado de:

https://www.unicef.org/socialpolicy/files/Human_Rights_and_Capabilities.pdf

Sen, A. (2011). La idea de la justicia. Buenos Aires: Taurus. 
Stiglitz, J., Sen, A. \& Fitoussi, J. (2009). Informe de la Comisión sobre la Medición del Desarrollo Económico y del Progreso Social. Recuperado de: http://www.palermo.edu/ Archivos_content/2015/derecho/pobreza_multidimensional/bibliografia/Biblio_adic5.pdf

UNESCO (2017). Informe de seguimiento de la educación en el mundo 2016. La educación al servicio de los pueblos y el planeta: creación de futuros sostenibles para todos. París: UNESCO. Recuperado de: http://unesdoc.unesco.org/images/0024/002485/248526S.pdf

Wolff, J. \& De-Shalit, A. (2007). Disadvantage. New York: Oxford University Press. 